\title{
Polo-like kinase 3 inhibits glucose metabolism in colorectal cancer by targeting HSP90/STAT3/HK2 signaling
}

\author{
Baochi Ou ${ }^{1+}$, Hongze Sun ${ }^{1 \dagger}$, Jingkun Zhao ${ }^{2}$, Zhuoqing $\mathrm{Xu}^{2}$, Yuan Liu ${ }^{1}$, Hao Feng ${ }^{2}$ and Zhihai Peng ${ }^{{ }^{*}}$
}

\begin{abstract}
Background: Polo-like kinase 3 (PLK3) has been documented as a tumor suppressor in several types of malignancies. However, the role of PLK3 in colorectal cancer (CRC) progression and glucose metabolism remains to be known.

Methods: The expression of PLK3 in CRC tissues was determined by immunohistochemistry. Cells proliferation was examined by EdU, CCK-8 and in vivo analyses. Glucose metabolism was assessed by detecting lactate production, glucose uptake, mitochondrial respiration, extracellular acidification rate, oxygen consumption rate and ATP production. Chromatin immunoprecipitation, luciferase reporter assays and co-immunoprecipitation were performed to explore the signaling pathway. Specific targeting by miRNAs was determined by luciferase reporter assays and correlation with target protein expression.
\end{abstract}

Results: PLK3 was significantly downregulated in CRC tissues and its low expression was correlated with worse prognosis of patients. In vitro and in vivo experiments revealed that PLK3 contributed to growth inhibition of CRC cells. Furthermore, we demonstrated that PLK3 impeded glucose metabolism via targeting Hexokinase 2 (HK2) expression. Mechanically, PLK3 bound to Heat shock protein 90 (HSP90) and facilitated its degradation, which led to a significant decrease of phosphorylated STAT3. The downregulation of p-STAT3 further suppressed the transcriptional activation of HK2. Moreover, our investigations showed that PLK3 was directly targeted by miR-106b at post-transcriptional level in CRC cells.

Conclusion: This study suggests that PLK3 inhibits glucose metabolism by targeting HSP90/STAT3/HK2 signaling and PLK3 may serve as a potential therapeutic target in colorectal cancer.

Keywords: Colorectal cancer, PLK3, Glucose metabolism, HSP90, STAT3, HK2

\section{Background}

Colorectal cancer (CRC) is one of the most common causes of tumor-related deaths worldwide with characteristics of unfavorable curative effect and poor prognosis [1]. Although progresses in screening and treatment have been made over the last decade, CRC remains a major health problem due to increasing morbidity and mortality [2]. Hence, elucidating the biologic characteristics and

\footnotetext{
*Correspondence: zhihai.peng@hotmail.com; m15021057659@163.com ${ }^{\dagger}$ Baochi Ou and Hongze Sun contributed equally to this work.

${ }^{1}$ Department of General Surgery, Shanghai General Hospital, Shanghai Jiao Tong University School of Medicine, No. 100, Haining Road, Shanghai 200080, China

Full list of author information is available at the end of the article
}

molecular mechanisms could be tremendously beneficial to the treatment of colorectal cancer.

To sustain continuous growth, cancer cells must reprogram canonical metabolic pathways to adapt to challenging hypoxic environments [3]. The Warburg effect, a core hallmark of cancer, is characterized by elevated glucose consumption and conversion of pyruvate to lactate even under normoxia [4]. Numerous evidences have revealed that activation of oncogenes or loss-of-function of tumor suppressors is responsible for aberrant metabolism in cancer cells [5]. For instance, the transcription factor c-Myc increases the expression of glycolytic genes, thereby promoting glycolysis and lactate secretion [6]. P53 helps cells adapt to limited periods of

(c) The Author(s). 2019 Open Access This article is distributed under the terms of the Creative Commons Attribution 4.0 International License (http://creativecommons.org/licenses/by/4.0/), which permits unrestricted use, distribution, and 
metabolic stress and resist the shift to glycolysis [7]. Although it is acknowledged that glycolysis occurs in CRC, the mechanism driving aerobic glycolysis remains largely unknown.

The Polo-like kinases (PLKs) are a family of highly conserved serine/threonine kinases, comprising five known members (PLK1-5). Each of them possesses a conserved $\mathrm{N}$-terminal kinase domain and one or more polo-box domains (PBDs) at the C-terminus [8]. PLKs have been shown to regulate a number of important cellular processes, such as cycle progression, mitosis and DNA damage response [9]. Moreover, our previous investigation suggested that PLK2 promoted tumor growth and restrained cells apoptosis, while PLK3 seemed to have tumor-suppressive feature in CRC [10]. Recently, several studies have demonstrated that PLKs play critical roles in energy metabolism. Li et al. find that the phosphorylation of PTEN by PLK1 contributes to a tumorpromoting metabolic state [11]. In addition, targeting PLK1 significantly alters various genes associated with a decrease of cellular metabolism [12]. However, little is known about the overall pathophysiological contribution of PLK3 to CRC malignancy and glucose metabolism.

In this study, we found that low expression of PLK3 in CRC tissues was correlated with worse prognosis of patients and PLK3 inhibited cancer cells growth. We further demonstrated that PLK3 suppressed glucose metabolism through downregulating Hexokinase 2 (HK2) expression in CRC. Mechanically, PLK3 interacted with Heat shock protein 90 (HSP90) and contributed to its degradation, which led to a significant reduction of phosphorylated STAT3. The downregulation of p-STAT3 further depressed the transcriptional activation of HK2 in the context of PLK3/HSP90 signaling. These findings indicate that the PLK3/HSP90/ STAT3/HK2 pathway serves as an important modulator of glucose metabolism in human colorectal cancer.

\section{Methods}

\section{Cells and reagents}

The human CRC cell lines and HEK293T were obtained from the American Type Culture Collection. SW480, SW620 and SW1116 are cultured in L-15 medium with $10 \%$ fetal bovine serum (FBS). HCT116, HT29 were maintained in McCoy' 5A medium with $10 \%$ FBS, and $\mathrm{CaCo} 2, \mathrm{RKO}$ and DLD1 were cultured in RPMI-1640 medium with the same components. Cells were detected to be free of mycoplasma using a Mycoplasma Detection Kit (Roche, USA). MG-132 (Sigma) was used to inhibit proteasome-mediated proteolysis in some experiments.

\section{Immunohistochemistry (IHC)}

The tissue microarray used in this study has been previously described [10]. Immunostaining was performed according to the manufacturer's protocol (Immunostain SP kit, DakoCytomation, USA). The results of IHC were determined by the percentage of positive cells and staining intensity (staining intensity: negative $=0$, weak $=1$, moderate $=2$, strong $=3$; and percentage of cells stained: $0=0-1 \%, \quad 1=1-5 \%, 2=6-29 \%, 3=30-59 \%, 4=60-$ $100 \%)$. These two values were multiplied together to generate a single score for each case. All cases were grouped as either negative (score $0-3$ ) or positive (score $>3$ ).

\section{Quantitative real-time PCR (qPCR) and PCR array}

Total RNA was extracted by using TRIzol reagent (Invitrogen). cDNA was synthesized with a TaKaRa PrimeScript RT reagent kit. The expression status of candidate genes and GAPDH were detected by using an ABI 7900HT RealTime PCR system (Applied Biosystems). All of the reactions were done in triplicate. Primer sequences are shown in Additional file 1: Table S1. In addition, we performed the human glucose metabolism $\mathrm{RT}^{2}$ profiler PCR array, which contained 84 key genes involved in glycolysis, TCA cycle, Pentose Phosphate Pathway and so on.

\section{Immunoblotting}

Immunoblotting was carried out as previously described [13]. Antibodies against PLK3 (4896), PCNA (2586), pSTAT3 (Tyr705, 9145), p-STAT3 (Ser727, 9134), p-p65 (3033) and total STAT3 (9139) were purchased from Cell Signaling Technology. Antibodies against HK2 (ab104836), HSP90 (ab13492), HIF-1 $\alpha$ (ab2185), c-Myc (ab32072), MZF1 (ab64866) and GAPDH (ab9484) were purchased from Abcam.

\section{Stable transfection and transfection of oligonucleotides} Lentiviral pGLV-PLK3 particles and shRNA plasmids targeting PLK3 mRNA were purchased from Genepharma (Shanghai, China). Lentivirus particles were transfected into the CRC cells in the presence of polybrene and selected using $5 \mu \mathrm{g} / \mathrm{ml}$ puromycin. All stable transfected cells were tested regularly by immunoblotting to ensure the efficiency of upregulation or downregulation. miRNA mimics were synthesized by Ambion (Austin, USA). Small interfering RNAs (siRNAs) targeting HK2, STAT3 and HSP90 were synthesized by RiboBio (Guangzhou, China). Cells were transfected with the oligonucleotides using Lipofectamine 3000 (Invitrogen) and harvested $48 \mathrm{~h}$ posttransfection.

\section{CCK-8 and 5-ethynyl-2'-deoxyuridine (EdU) assay}

CCK-8 proliferation assay was conducted as previously reported [14]. In addition, cells were incubated with EdU at a final concentration of $10 \mu \mathrm{M}$ for $2 \mathrm{~h}$ and analyzed using the Click-iT EdU Assay (Roche, USA) according to the 
instructions. Images were captured under microscope and the percentage of EdU-positive cells was calculated.

\section{Glycolysis analysis and ATP production}

Lactate Colorimetric Assay Kits (Biovision) and Glucose Uptake Colorimetric Assay Kits (Biovision) were used to examine the glycolysis process in cancer cells, according to the manufacturer's instructions. The glycolytic capacity was examined by using the Glycolysis Stress Test Kit. ATP assay kit (Promega) was used to detect ATP production in tumor cells.

\section{Oxygen consumption rate (OCR) and extracellular acidification rate (ECAR)}

ECAR and OCR were determined by the Bioscience XF96 Extracellular Flux Analyzer. Briefly, seeded onto 96-well plates and cultured overnight, cells were then washed with Seahorse buffer (Dulbecco's modified Eagle's medium with phenol red containing glucose, sodium pyruvate and glutamine). Later, $175 \mu \mathrm{L}$ of Seahorse buffer plus $25 \mu \mathrm{L}$ each of oligomycin, FCCP, and rotenone were automatically added to measure the OCR. The ECAR was calculated by injecting $25 \mu \mathrm{L}$ each of glucose, oligomycin, and 2-deoxy-glucose (2-DG) into cells. The values of OCR and ECAR were normalized to the number of cells per well and are shown as the mean $\pm \mathrm{SD}$.

\section{Chromatin immunoprecipitation (CHIP)}

ChIP assays were performed as previously described [15]. The chromatin immunoprecipitation Kit (Millipore) was used according to the manufacturer's instructions. The presence of predicted transcription factor binding regions pulled by antibodies was examined by qPCR. Primers to detect gene promoter occupancy were shown in Additional file 1: Table S1.

\section{Luciferase reporter assay}

The HK2 promoter was cloned into the pGL3-Basic luciferase plasmid (Promega, Madison, USA) to construct WT $P_{\text {HK2 }}$ reporter (Fig. 4d). A 9-bp sequence as the putative STAT3 binding site was deleted in the Mut $P_{\mathrm{HK} 2}$ reporter (Fig. 4d) Tumor cells were then cotransfected with WT $P_{\mathrm{HK} 2}$ or Mut $P_{\mathrm{HK} 2}$ constructs. The primers were present in Additional file 1: Table S1. For PLK3 3'-UTR-Renilla luciferase reporter assay, each reporter construct was co-transfected into HEK293T cells together with luciferase plasmid pGL3 and miR106 b mimics or Control RNA. After $48 \mathrm{~h}$ of incubation, luciferase activities were measured using the Dual luciferase Reporter Assay System (Promega).

\section{Co-immunoprecipitation}

Total cell lysates of cells were obtained in immunoprecipitation buffer, and then were incubated with anti-HSP90 antibody or the relative IgG control. The incubation was shaken on a rotating shaker for $2 \mathrm{~h}$ at $4{ }^{\circ} \mathrm{C}$. Then, the proteins were immunoprecipitated by protein A/G Sepharose (Santa Cruz). Beads were collected and loading buffer was added to boil with them. The supernatants were then detected by immunoblotting.

\section{Xenograft model}

Forty 4-week-old male BALB/c nude mice were randomly divided into 4 groups (10 for each group). One million tumor cells in $100 \mu \mathrm{l}$ PBS were injected subcutaneously. Tumor sizes were measured every 6 days and calculated as: $\mathrm{V}=\left(\mathrm{Width}^{2} \times\right.$ Length $^{2} / 2$. Xenografts were collected at 36th day for protein extraction. All experiments were conducted with the approval and guidance of the Animal Ethics Committee (Shanghai Jiao Tong University School of Medicine).

\section{Statistical analysis}

The data are presented as the means \pm SD of at least three independent experiments. Two-tailed unpaired Student $t$ tests and one-way analysis of variance were used for the data analysis. Kaplan-Meier method was used to assess patients' survival outcome. Differences were considered significant at * $P<0.05 ;{ }^{* *}, P<0.01$; and ***, $P<0.001$.

\section{Results}

\section{Clinical significance of PLK3 in colorectal cancer}

Our previous research has showed that PLK3 is lowly expressed in CRC tissues relative to matched normal tissues [10]. In this study, we further analyzed PLK3 expression in a tissue microarray containing 116 pairs of cancerous and matched normal tissue by IHC (Fig. 1a). Moreover, the correlation between pathologic factors and PLK3 expression were compared. In these cases, the negative expression of PLK3 was detected in 72 (62.1\%) of the tumor tissues, whereas $49(42.2 \%)$ of the adjacent normal specimens showed a negative signal (Table 1). Furthermore, a significant association was observed between the PLK3 negative group and positive group in tumor size $(P=0.008)$, lymphatic metastasis $(P=0.027)$ and TNM stage $(P=0.019)$ (Table 1$)$. Kaplan-Meier analysis revealed that patients in the PLK3 ${ }^{\text {negative }}$ group had a significantly poorer overall survival than those in the PLK3 ${ }^{\text {positive }}$ group $(P=0.013$; Fig. $1 b)$. In addition, PLK3 ${ }^{\text {negative }}$ patients had a shorter disease-free survival $(P<0.001$; Fig. 1c). However, multivariate analyses indicated that PLK3 expression was not an independent prognostic factor for survival in CRC patients (data not shown). 

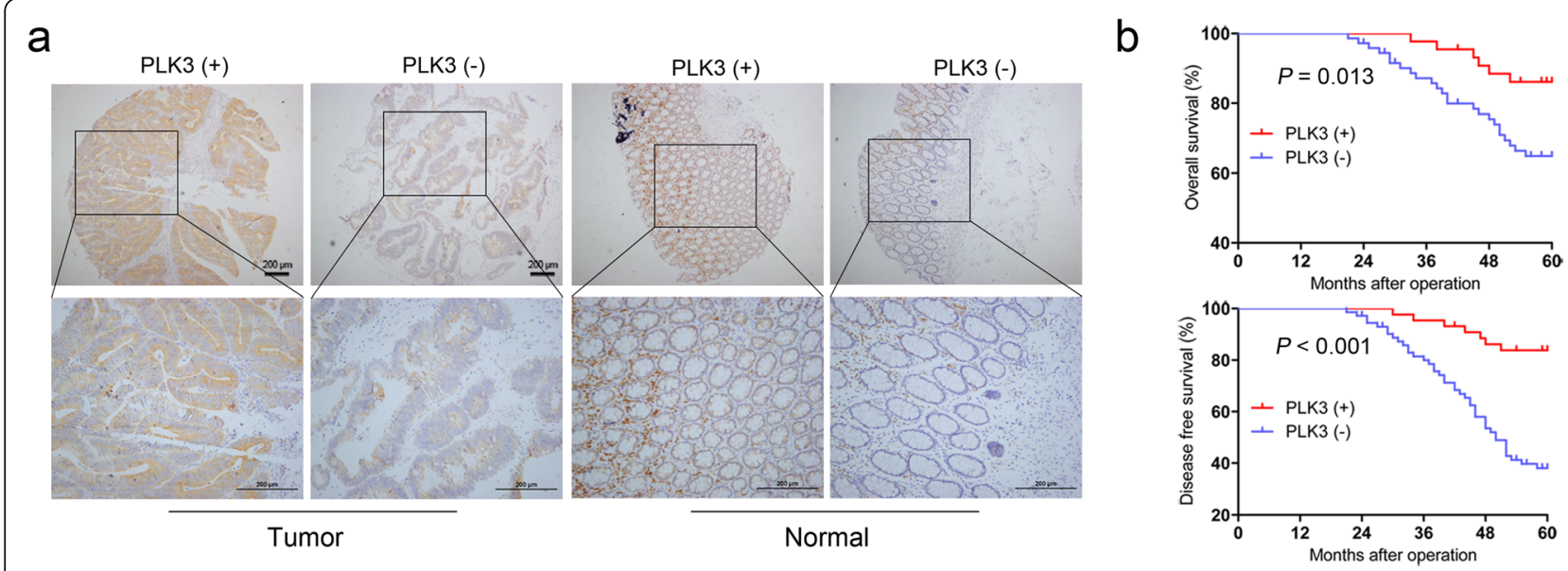

Fig. 1 Clinical significance of PLK3 in CRC patients. a PLK3 expression level in tumor tissues and the paired normal tissues was evaluated by IHC in the tissue microarray. $\mathbf{b}$ CRC patients with negative expression of PLK3 presented worse overall survival, and disease-free survival compared with that of positive expression of PLK3

\section{PLK3 inhibits proliferation and glucose metabolism of CRC cells}

The expression of PLK3 in eight CRC cell lines was detected. As shown in Additional file 2: Figure S1a and b, PLK3 was differently expressed and especially high in HCT116 and HT29, but low in SW480 and RKO. Thus, we determined to knock down PLK3 in HCT116 cells, and selected SW480 for exogenous PLK3 overexpression. The efficiency of transfection was confirmed by immunoblotting (Additional file 2: Figure S1c). PLK3 overexpression or silencing exert no influence on the expression of other PLK members (Additional file 2: Figure S1d). We then performed EdU incorporation to examine the effect of altering PLK3 levels on cells proliferation. As shown in Fig. 2a, PLK3 overexpression suppressed proliferative ability of SW480 cells and PLK3 knockdown promoted HCT116 cells proliferation. In addition, CCK- 8 assay also confirmed the effect of PLK3 on proliferation of CRC cells (Fig. 2b). To study the effects of PLK3 in vivo, we subcutaneously injected nude mice with tumor cells. As expected, PLK3 overexpression inhibited tumor growth and reduced tumor weight in the xenograft mouse model, whereas PLK3 knockdown had the opposite effects (Fig. 2c). Subsequent immunoblotting analysis demonstrated that PCNA (marker of proliferation) expression was significantly influenced by PLK3 in tissues from xenograft tumors (Fig. 2d).

To determine whether cell proliferation mediated by PLK3 reflects a change in glucose metabolism, we first examined lactate production and glucose uptake, two primary indicators of the Warburg effect in tumor cells. As shown in Fig. 2e, ectopic expression of PLK3 reduced lactate production and glucose uptake of SW480 cells, while silencing PLK3 increased lactate production and glucose uptake of HCT116 cells. We then detected the
ECAR, which reflects the lactate-induced acidification of the medium surrounding cells. The data indicated that the ECAR was increased in PLK3-silencing cells, whereas PLK3 overexpression decreased the ECAR value and may play a suppressive role in lactate formation (Fig. 2f). Additionally, OCR, the indicator of mitochondrial respiratory capacity, was measured in HCT116 and SW480 cells. Interestingly, PLK3-overexpressing cells exhibited lower OCR relative to the control. Conversely, the OCR was increased in PLK3-depleted HCT116 cells (Fig. 2g). ATP is indispensable to rapid proliferation and metastasis of cancer cell. Herein, we found that PLK3 overexpression exerted an inhibitory effect on ATP production, while PLK3 knockdown increased ATP levels in HCT116 cells (Fig. 2h). Altogether, these findings suggest PLK3 plays a critical role in proliferation and glucose metabolism of CRC cells.

\section{HK2 is responsible for PLK3-mediated CRC cells glycometabolism}

To elucidate the molecular mechanisms for PLK3mediated glycometabolism, we analyzed differentially expressed genes for cancer cells using a human glucose metabolism PCR array. Among 84 genes examined, several genes were significantly upregulated in cells with low expression of PLK3 (SW480, HCT116/sh-PLK3) relative to their controls (Fig. 3a). HK2, an enzyme that catalyzes the first and irreversible step of glycolysis, was selected for further investigation because it was the most upregulated gene. Indeed, immunoblotting analysis confirmed that PLK3 overexpression reduced HK2 protein expression in SW480 and that PLK3 knockdown improved the levels of this protein in HCT116 cells (Fig. 3b). To further explore whether HK2 is crucial for PLK3-mediated cell glycolysis, siRNAs were used to 
Table 1 Relationship between PLK3 expression and clinicopathologic variables in 116 CRC patients

\begin{tabular}{|c|c|c|c|c|}
\hline \multirow[t]{2}{*}{ Variable } & \multirow{2}{*}{$\begin{array}{l}\text { Case } \\
(n=116)\end{array}$} & \multicolumn{2}{|c|}{ PLK3 expression } & \multirow[t]{2}{*}{$P$ value } \\
\hline & & Negative & Positive & \\
\hline Tissues & & & & 0.003 \\
\hline Normal tisssues & 116 & 49 & 67 & \\
\hline Carcinoma & 116 & 72 & 44 & \\
\hline Gender & & & & 0.936 \\
\hline Male & 68 & 42 & 26 & \\
\hline Female & 48 & 30 & 18 & \\
\hline Age & & & & 0.623 \\
\hline$\leq 65$ & 52 & 31 & 21 & \\
\hline$>65$ & 64 & 41 & 23 & \\
\hline Location & & & & 0.301 \\
\hline Left hemicolon & 12 & 10 & 2 & \\
\hline Right hemicolon & 33 & 22 & 11 & \\
\hline Sigmoid colon & 24 & 14 & 10 & \\
\hline Rectum & 47 & 26 & 21 & \\
\hline Tumor size (cm) & & & & 0.008 \\
\hline$\leq 4 \times 3$ & 53 & 26 & 27 & \\
\hline$>4 \times 3$ & 63 & 46 & 17 & \\
\hline \multicolumn{5}{|l|}{ Tumor histology } \\
\hline Tubular & 98 & 60 & 38 & 0.796 \\
\hline Mucinous & 16 & 11 & 5 & \\
\hline Papillary & 2 & 1 & 1 & \\
\hline Extent of invasion & & & & 0.332 \\
\hline $\mathrm{T} 1+\mathrm{T} 2$ & 31 & 17 & 14 & \\
\hline $\mathrm{T} 3+\mathrm{T} 4$ & 85 & 55 & 30 & \\
\hline Lymphatic metastasis & & & & 0.027 \\
\hline No & 56 & 29 & 27 & \\
\hline $\mathrm{N} 1+\mathrm{N} 2$ & 60 & 43 & 17 & \\
\hline Metastasis & & & & 0.444 \\
\hline MO & 105 & 64 & 41 & \\
\hline M1 & 11 & 8 & 3 & \\
\hline TNM stage & & & & 0.019 \\
\hline $1+\|$ & 55 & 28 & 27 & \\
\hline$I I I+I V$ & 61 & 44 & 17 & \\
\hline \multicolumn{5}{|l|}{ CEA level } \\
\hline$<5.0$ & 89 & 54 & 35 & 0.574 \\
\hline$\geq 5.0$ & 27 & 18 & 9 & \\
\hline
\end{tabular}

knockdown HK2 expression in cells with low expression of PLK3 (SW480 and HCT116/sh-PLK3), whose effects were confirmed by immunoblotting (Fig. 3c and Additional file 3: Figure S2a). After si-HK2 treatment, the increased glucose uptake and lactate production induced by PLK3 silencing were dramatically abolished in tumor cells (Fig. 3d). Similarly, the values of ECAR (Fig. 3e) and OCR (Fig. 3f) were decreased remarkably after HK2 silencing in tumor cells. ATP production was also suppressed upon HK2 knockdown in SW480 and HCT116/sh-PLK3 cells (Fig. 3g).

We then analyzed HK2 expression in the same tissue microarray (Representative images: Fig. 3h). As expected, HK2 was highly expressed in tumor samples compared to normal samples $(67.2 \%$ versus $44.8 \%, P=0.001$, Additional file 1: Table S2). Furthermore, our results showed a negative association between PLK3 and HK2 expression (Pearson's correlation, $r=-0.346, P<0.001$, Fig. 3i) in CRC tissues. These data indicate that PLK3 plays a role in CRC glucose metabolism by regulating HK2 expression.

\section{PLK3-mediated STAT3 transcriptionally regulates HK2 expression in CRC cells}

To understand how PLK3 suppresses HK2 gene expression in CRC cells, we hypothesized that HK2 transcription was mediated by one or more transcription factors chaperoned by PLK3. We performed a literature search and shortlisted five proteins that have been reported to regulate HK2 transcription in human cells, namely, HIF$1 \alpha$, с-Myс, STAT3, NF-кB, Myeloid Zinc Finger-1 (MZF1) [16-20]. The protein levels of these transcription factors were then examined. As indicated in Fig. 4a, ectopic expression of PLK3 led to downregulation of $\mathrm{p}$ STAT3 (Ser727), while silencing PLK3 dramatically activated p-STAT3 (Ser727) expression. The expression of other proteins (Fig. 4a) exhibited no significant changes. It is known that the transcriptional activation of STAT3 depends on its phosphorylation at tyrosine 705 or serine 727 residue, which leads to different functions [21]. Indeed, when using siRNAs to knockdown pSTAT3 (Ser727) in SW480 and HCT116/sh-PLK3 cells, we confirmed that blockade of p-STAT3 (Ser727) significantly suppressed HK2 expression levels (Fig. 4b).

Next, we used TransFac and Genomatix software [22] to analyze the upstream sequences of $\mathrm{HK} 2$, ranging from -2000 to +200 bp relative to the transcription start site. Only one putative binding site was identified within the human HK2 promoter region. We then constructed luciferase reporters under control of either the wild-type HK2 promoter or a mutant promoterwith a deletion of the 9-bp STAT3 binding site (named as WT $P_{\mathrm{HK} 2}$ reporter or Mut $P_{\mathrm{HK} 2}$ reporter, respectively). The results demonstrated that this site was critical for STAT3induced HK2 activation (Fig. 4c and d). Subsequent CHIP assays also verified the direct binding of STAT3 to the predicted site of HK2 promoter in CRC cells and tissues (Fig. 4e). Altogether, these findings suggest that STAT3 is a direct transcriptional activator for HK2 in human colorectal cancer. 


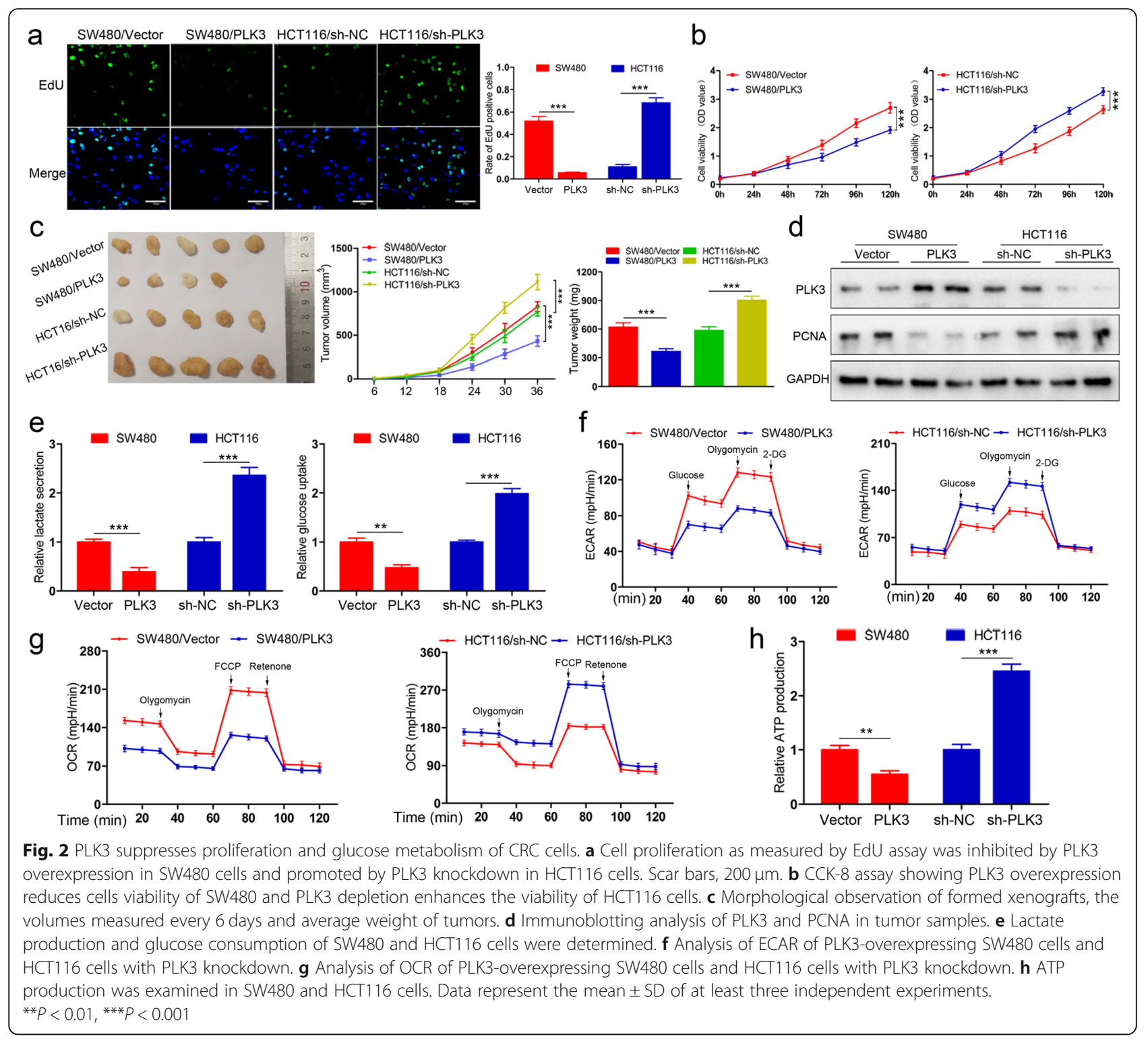

\section{HSP90 stabilization is involved in PLK3-mediated CRC glucose metabolism}

Since the direct interaction of PLK3 and STAT3 was not observed in CRC cells (data not shown), the mechanism that bridges these two molecules was further explored. Previous researches have demonstrated that PLK3 may play a role in oncogenesis by mediating various substrates, such as PTEN, HIF-1 $\alpha$, HSP90 and so on [23-25]. In this study, we screened the expression of these substrates and found that HSP90 was significantly influenced by PLK3 overexpression or silencing (Fig. 5a), whereas HSP90 mRNA levels varied slightly (Fig. 5b). The stability of HSP90 could be rescued by MG-132 treatment, suggesting the regulation between PLK3 and HSP90 involves phosphorylation-mediated degradation by proteasome (Fig. 5a). Moreover, when using siRNAs to knockdown
HSP90 expression in the cells with low expression of PLK3, we found that si-HSP90 significantly suppressed the expression of p-STAT3 (Ser727) and HK2 (Fig. 5c). In addition, co-immunoprecipitation assays showed that both PLK3 and STAT3 were detected in the immunoprecipitatation products (Fig. 5d and Additional file 3: Figure S2b). This confirmed the interactions between PLK3, STAT3 and HSP90, which were significantly inhibited when cells were transfected with si-HSP90 (Fig. 5d and Additional file 3: Figure S2b). Subsequent experiments demonstrated that silencing HSP90 impeded lactate production, glucose uptake, mitochondrial respiration, ECAR, OCR and ATP production in SW480 and HCT116/sh-PLK3 cells (Fig. 5e-h). Thus, our data indicate that PLK3-medatied HSP90 plays a critical role in glucose metabolism of CRC cells. 


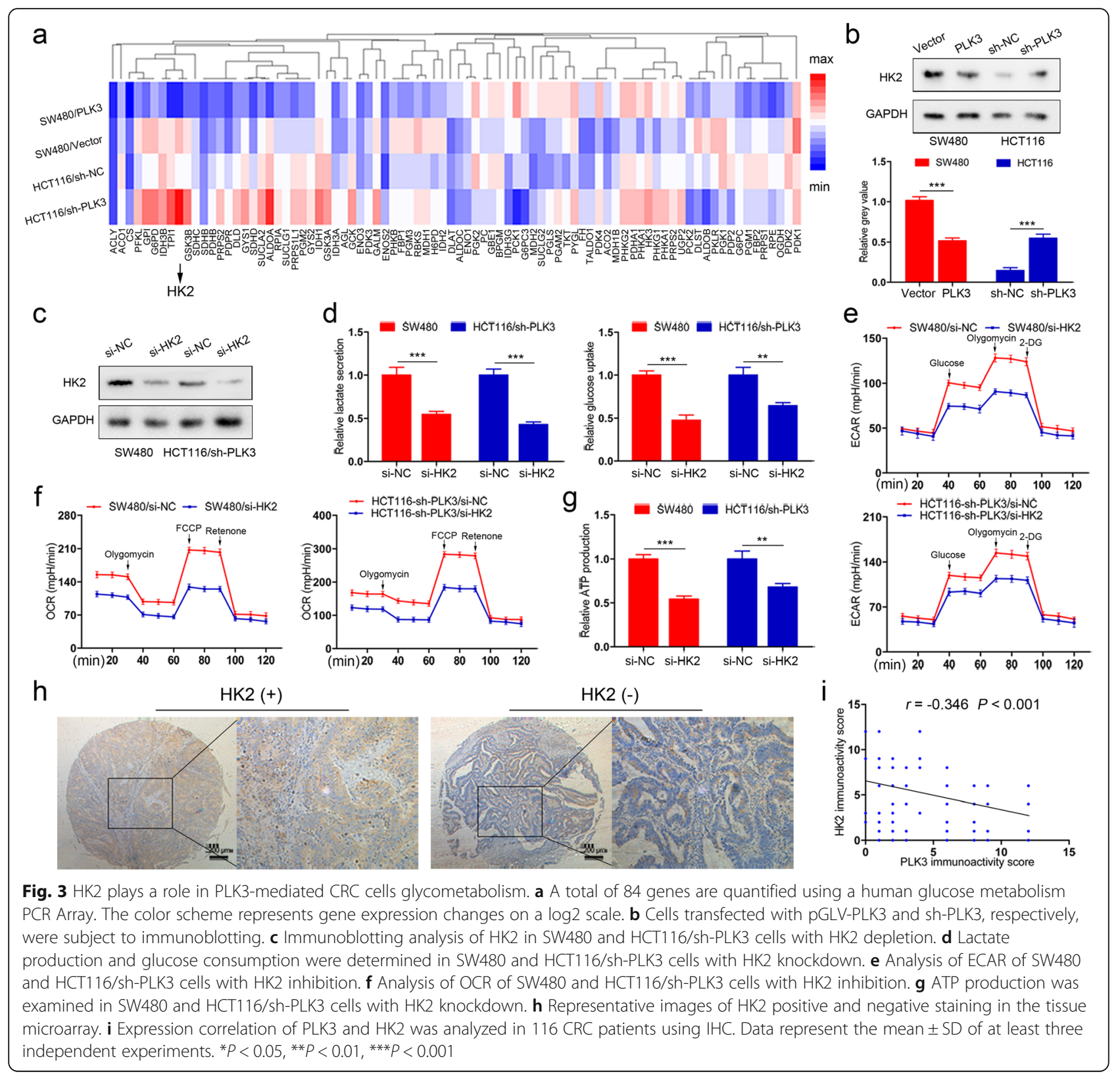

\section{PLK3 expression is directly suppressed by miR-106b in CRC cells}

MicroRNAs (miRs) are small, noncoding RNAs that coordinate the gene expression at the post-transcriptional level [26]. To understand the mechanism responsible for the low expression of PLK3 in CRC, we hypothesized that PLK3 was directly regulated by microRNAs. The Cancer Genome Atlas (TCGA) was screened for miRs that were significantly dysregulated in CRC (fold change $\geq 2$ or $\leq 0.5$ ). Candidate targets were searched using the prediction tools including miRanda, miRWalk and TargetScan. 5 microRNAs were finally identified and selected for further investigation (Fig. 6a). Interestingly, only miR-106b mimics-transfected cells exhibited a significant decrease in the mRNA expression of PLK3 (Fig. 6b). Indeed, ectopic expression of miR-106b decreased PLK3 protein levels in cells with high PLK3 expression (SW480/PLK3 and HCT116), whereas inhibition of miR-106b by anti-miR-106b led to a converse effect (Fig. 6c).

To confirm the regulation of PLK3 by miR-106b, we constructed the luciferase reporters containing the wildtype (WT) or mutated 3'-UTR sequences of PLK3 (Fig. 6d). The reporters were co-transfected with miR106b mimics or negative control RNAs into HEK293T cells. Interestingly, we observed that the luciferase activity of wild-type vector, but not its mutant counterpart, was significantly suppressed by miR-106b (Fig. 6e). 


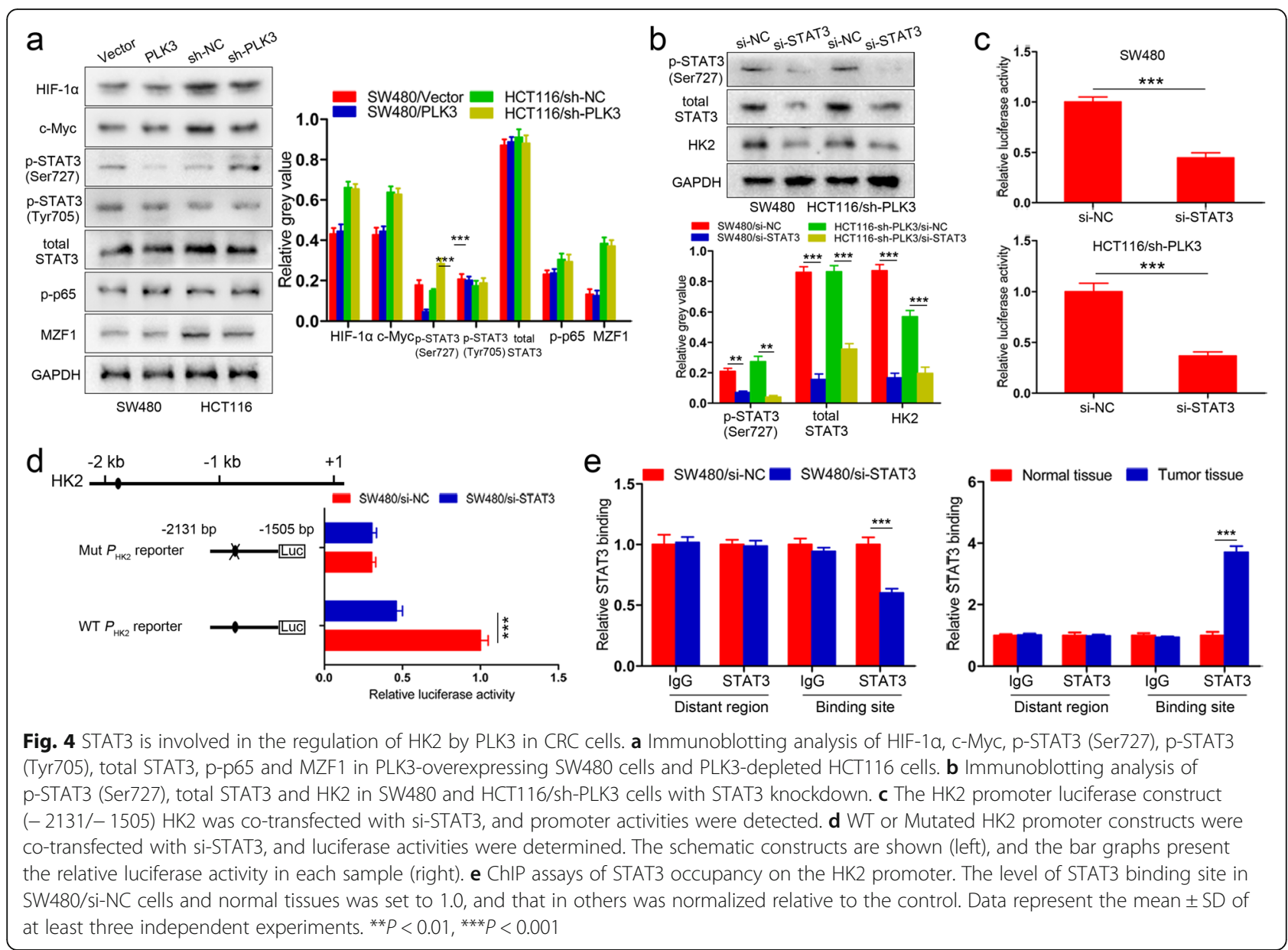

Collectively, the schematic diagram of our study is shown in Fig. $6 f$.

\section{Discussion}

In recent decades, the Warburg effect has been highlighted as a hallmark of cancer [27]. Even in the presence of an oxygen supply, tumor cells preferentially utilize glycolysis rather than mitochondrial oxidative phosphorylation to produce ATP for biosynthesis. The reprogramming of tumor metabolism is suggested to be controlled by various oncogenic signals $[28,29]$. In colorectal cancer, the pro-inflammatory cytokines TNF $\alpha$ and IL-17 have been reported to cooperatively stimulate glycolysis [30]. The study by Tambe et al. suggests the tumor suppressor drs inhibits Warburg effect via lactate dehydrogenase-B [31]. Furthermore, Che-1 mediates HIF- $1 \alpha$ stabilization to affect glucose metabolism in the response to hypoxia [32].

PLKs are dysregulated in many types of cancer, such as liver cancer, brain cancer and ovarian cancer [9]. To date, only PLK1 was identified as an important coordinator of glucose metabolism in tumor cells $[11,12]$. The roles of PLK2-5 in cancer glucose metabolism remain to be elucidated. Herein, we found that PLK3 was lowly expressed in $62.1 \%$ of tumor samples examined, suggesting that alteration of PLK3 level is a frequent event in human CRC. Moreover, a remarkable correlation between PLK3 expression and tumor size, lymphatic metastasis and TNM stage was found. PLK3 negative expression was significantly associated with poor prognosis of CRC patients. Subsequent in vitro functional assays demonstrated that PLK3 inhibited CRC cells proliferation. In vivo experiments also supported a tumor-suppressive function of PLK3 in CRC. Thus, our investigations focused on the role and molecular mechanism of PLK3 in CRC glucose metabolism.

Next, a series of aerobic glycolysis-related assays were performed. We found that overexpression of PLK3 dramatically inhibited glycolysis, whereas silencing PLK3 promoted glucose metabolism in CRC cells. To explore the potential mechanism, we profiled the differentially expressed genes involved in glycolysis, tricarboxylic acid cycle, pentose phosphate pathway and so on. HK2 was identified as a candidate target and chosen for further exploration. HK2, the major isozyme of hexokinase, catalyzes the first irreversible step in the glycolytic pathway 


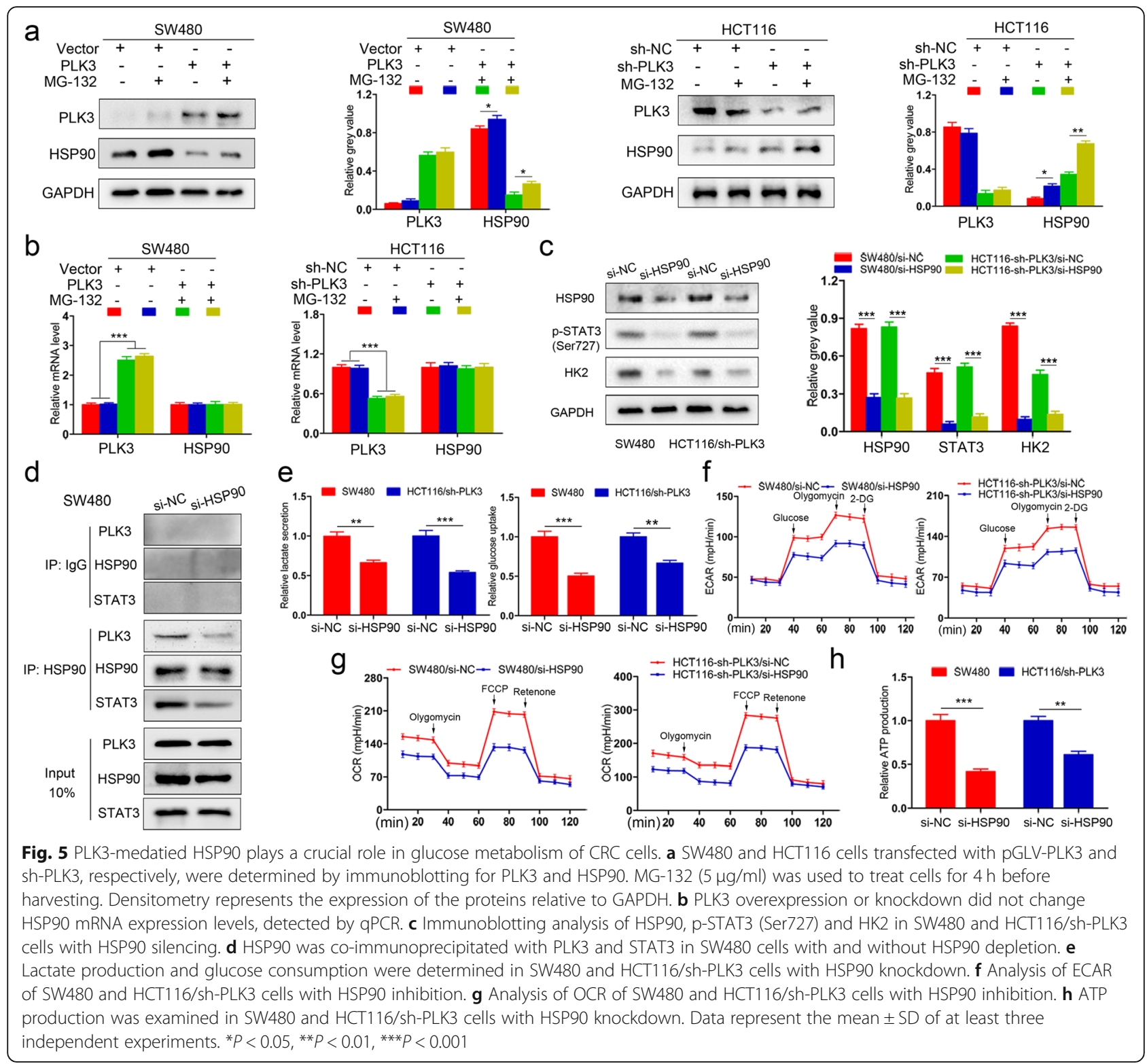

by phosphorylating glucose to G6P [33]. It has been reported that HK2 is exclusively upregulated in many types of cancers and plays a key role in the Warburg effect [34]. In this study, HK2 expression level was found to be decreased under condition of PLK3 overexpression, while silencing PLK3 increased HK2 expression in tumor cells. Inhibition of HK2 significantly suppressed glucose metabolism of PLK3-depleted CRC cells. Furthermore, PLK3 was inversely associated with HK2 protein expression in CRC tissues. These data provide clues on the mechanism that PLK3 might impede CRC glycolysis by targeting HK2.

We then explored the potential signal pathways implicated in the regulation of HK2 expression. A comprehensive literature search was performed and we shortlisted five transcription factors that have been reported to regulate HK2 transcription in human cells. The results showed that p-STAT3 (Ser727) expression was significantly inhibited by PLK3 overexpression, while PLK3 depletion increased p-STAT3 (Ser727) protein levels. Moreover, CHIP and luciferase reporter assays demonstrated that STAT3 directly bound to the promoter region of HK2 and contributed to its expression. Recently, STAT3 signaling has been shown to reprogram cellular metabolism in human malignancies. The study by Lin et al. [35] indicates that palmitic acid limits glycometabolism through the attenuation of STAT3 pathway in hepatocellular carcinoma. Furthermore, STAT3 signaling participates in the pyruvate kinase isoenzyme type M2-mediated glycolysis of breast cancer cells [36]. Our data herein suggested STAT3 


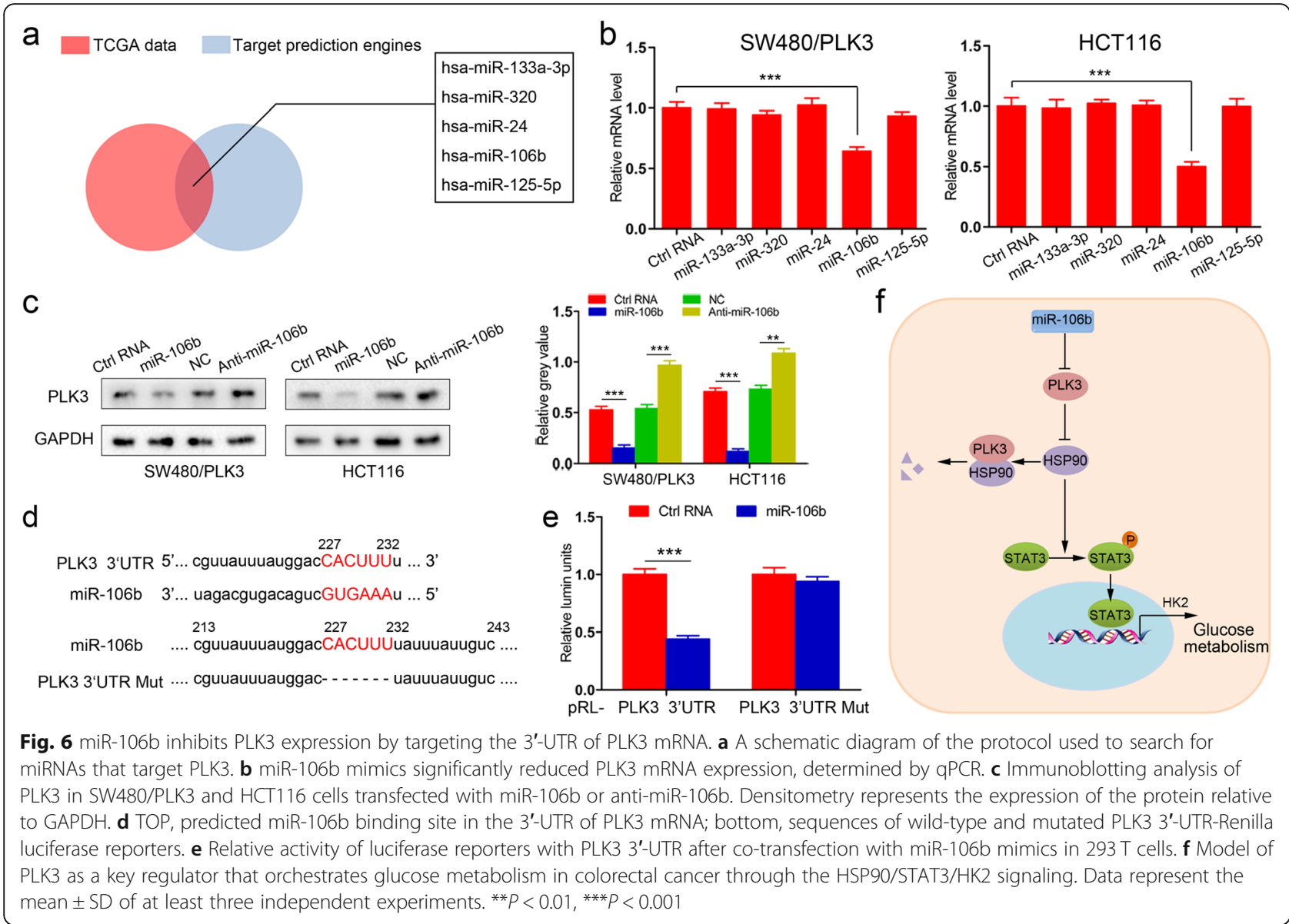

played a crucial role in HK2 expression and glucose metabolism of human CRC cells. However, we could not exclude the possibility that other forms of STAT3 phosphorylation may be involved in HK2 downregulation.

It has been recognized that the PBDs located in the Cterminal region of PLKs are responsible for their functions. The PBD acts as a key mediator via phosphorylationdependent interactions with downstream targets [8]. Previous researches have revealed that PLK3 plays a role in carcinogenesis by regulating various substrates [37]. In this study, we screened the expression of these targets and found that HSP90 was significantly influenced by PLK3 silencing or overexpression. The regulation of HSP90 by PLK3 involves proteasome-mediated degradation. Moreover, using siRNAs to target HSP90, we observed that the expression levels of p-STAT3 (Ser727) and HK2 were significantly downregulated. In addition, the interactions between PLK3, STAT3 and HSP90 were validated by coimmunoprecipitation in CRC cells. These findings suggest PLK3 directly binds to HSP90 and contributes to the following degradation of HSP90 and inactivation of STAT3. Subsequent experiments further showed that HSP90 inhibition suppressed glucose metabolism in the PLK3-depleted cells. Altogether, our investigations demonstrate that
PLK3/HSP90/STAT3/HK2 signaling plays a critical role in glycometabolism of CRC cells.

Emerging evidence indicates that microRNAs have important functions during tumor progression through posttranscriptional regulation of gene expression [26]. Furthermore, microRNAs have also been documented as crucial mediators in the reprogramming of cellular energy metabolism [38]. In this study, we combined the data from TCGA as well as microRNAs prediction engines and identified miR-106b as the upstream regulator of PLK3 in CRC cells. Luciferase reporter assays also confirmed that PLK3 was a direct target of miR-106b in HEK293T cells. Previously, miR-106b has been reported as a contributor for CRC cell migration and invasion [39]. Moreover, miR-106b could enhance CRC cell radioresistance and tumor-initiating capacity [40]. Our results herein suggested miR-106b may have a function through targeting PLK3 in CRC cells. However, the role of miR-106b in cancer glucose metabolism remains to be clarified.

\section{Conclusions}

In conclusion, the present study demonstrates that PLK3 is frequently downregulated in tumor tissues compared 
to normal tissues. Low expression of PLK3 is correlated with poor survival of CRC patients. Moreover, we unravel a novel mechanism that PLK3 suppresses cells glucose metabolism via the HSP90/STAT3/HK2 pathway. PLK3 expression is directly suppressed by miR106b in CRC cells. These findings point to a critical role of PLK3 in the colorectal cancer glycometabolism, which might provide a promising therapeutic target for the treatment of CRC.

\section{Supplementary information}

Supplementary information accompanies this paper at https://doi.org/10. 1186/s13046-019-1418-2

Additional file 1: Table S1. Primer sequences used in this study. Additional file 2: Figure S1. a PLK3 expression in eight CRC cell lines was tested by qPCR. b PLK3 expression in eight CRC cell lines was detected by immunoblotting. Densitometry represents the expression of the proteins relative to GAPDH. c SW480 and HCT116 cells transfected with pGLV-PLK3 and sh-PLK3, respectively, were subject to immunoblotting. Densitometry represents the expression of the proteins relative to GAPDH. d The relative mRNA expression of PLK1, PLK2, PLK4 and PLK5 in the cells with PLK3 silencing or overexpression.

Additional file 3: Figure S2. a Immunoblotting analysis verifying HK2 knockdown in SW480 and HCT116/sh-PLK3 cells. Densitometry represents the expression of the proteins relative to GAPDH. b Quantitative analysis of PLK3/HSP90 and HSP90/STAT3 interaction.

\section{Abbreviations}

ChIP: Chromatin immunoprecipitation; CRC: Colorectal cancer; HIF1a: Hypoxia-inducible factor 1a; HK2: Hexokinase 2; HSP90: Heat shock protein 90; IHC: Immunohistochemistry; miRNAs: microRNAs; MZF1: Myeloid zinc finger 1; NF-kB: Nuclear factor KB; PLK: Polo-like kinase; qPCR: Quantitative real-time PCR; siRNAs: Small interfering RNAs; STAT3: Signal transducer and activator of transcription

\section{Acknowledgements}

This work was supported by the National Natural Science Foundation of China (Grant No. SQ2017ZX106050, 81530044, 81202609).

\section{Authors' contributions}

Study conception and design: ZP, BO, HS. Data acquisition: BO, HS, ZX, JZ, YL, HF. Data Analysis and interpretation: ZX, JZ, YL, HF. Writing of the manuscript: $\mathrm{BO}, \mathrm{HS}$. All authors read and approved the final manuscript.

\section{Funding}

See acknowledgements.

\section{Availability of data and materials}

The dataset supporting the conclusions of this article is included within the article.

\section{Ethics approval and consent to participate}

All the experiments involving in human specimens and animals were in accordance with the ethical code and recommendation issued by Ethics Committee of Human Experimentation and Chinese Animal Community and with the Helsinki Declaration of 1975, as revised in 2008.

\section{Consent for publication}

All authors consent for publication.

\section{Competing interests}

The authors declare that they have no competing interests.

\section{Author details}

'Department of General Surgery, Shanghai General Hospital, Shanghai Jiao Tong University School of Medicine, No. 100, Haining Road, Shanghai 200080, China. ${ }^{2}$ Department of General Surgery, Ruijin Hospital, Shanghai Jiao Tong University School of Medicine, Shanghai, China.

Received: 15 July 2019 Accepted: 9 September 2019

Published online: 26 October 2019

\section{References}

1. Siegel RL, Miller KD, Jemal A. Cancer statistics, 2016. CA Cancer J Clin. 2016; 66:7-30.

2. Markowitz SD, Bertagnolli MM. Molecular origins of cancer: molecular basis of colorectal cancer. N Engl J Med. 2009;361:2449-60.

3. Koppenol WH, Bounds PL, Dang CV. Otto Warburg's contributions to current concepts of cancer metabolism. Nat Rev Cancer. 2011;11:325-37.

4. Cantor JR, Sabatini DM. Cancer cell metabolism: one hallmark, many faces. Cancer Discov. 2012;2:881-98.

5. Vander Heiden MG, Cantley LC, Thompson CB. Understanding the Warburg effect: the metabolic requirements of cell proliferation. Science. 2009;324: 1029-33.

6. Dang CV. MYC on the path to cancer. Cell. 2012;149:22-35.

7. Cheung EC, Vousden $\mathrm{KH}$. The role of p53 in glucose metabolism. Curr Opin Cell Biol. 2010;22:186-91.

8. Archambault V, Glover DM. Polo-like kinases: conservation and divergence in their functions and regulation. Nat Rev Mol Cell Biol. 2009;10:265-75.

9. de Carcer G, Manning G, Malumbres M. From Plk1 to Plk5: functional evolution of polo-like kinases. Cell Cycle. 2011;10:2255-62.

10. Ou B, Zhao J, Guan S, Wangpu X, Zhu C, Zong Y, Ma J, Sun J, Zheng M, Feng $\mathrm{H}$, Lu A. Plk2 promotes tumor growth and inhibits apoptosis by targeting Fbxw7/cyclin E in colorectal cancer. Cancer Lett. 2016;380:457-66.

11. Li Z, Li J, Bi P, Lu Y, Burcham G, Elzey BD, Ratliff T, Konieczny SF, Ahmad N, Kuang S, Liu X. Plk1 phosphorylation of PTEN causes a tumor-promoting metabolic state. Mol Cell Biol. 2014;34:3642-61.

12. Gutteridge RE, Singh CK, Ndiaye MA, Ahmad N. Targeted knockdown of polo-like kinase 1 alters metabolic regulation in melanoma. Cancer Lett. 2017:394:13-21.

13. Zhao J, Ou B, Han D, Wang P, Zong Y, Zhu C, Liu D, Zheng M, Sun J, Feng $\mathrm{H}$, Lu A. Tumor-derived CXCL5 promotes human colorectal cancer metastasis through activation of the ERK/Elk-1/Snail and AKT/GSK3beta/ beta-catenin pathways. Mol Cancer. 2017;16:70.

14. Xu Z, Zhu C, Chen C, Zong Y, Feng H, Liu D, Feng W, Zhao J, Lu A. CCL19 suppresses angiogenesis through promoting miR-206 and inhibiting Met/ ERK/Elk-1/HIF-1alpha/NEGF-A pathway in colorectal cancer. Cell Death Dis. 2018;9:974.

15. Ou B, Zhao J, Guan S, Feng H, Wangpu X, Zhu C, Zong Y, Ma J, Sun J, Shen $X$, Zheng M, Lu A. CCR4 promotes metastasis via ERK/NF-kappaB/MMP13 pathway and acts downstream of TNF-alpha in colorectal cancer. Oncotarget. 2016;7:47637-49.

16. Zhou L, Wang Y, Zhou M, Zhang Y, Wang P, Li X, Yang J, Wang H. HOXA9 inhibits HIF-1alpha-mediated glycolysis through interacting with CRIP2 to repress cutaneous squamous cell carcinoma development. Nat Commun. 2018:9:1480.

17. Yu P, Wilhelm K, Dubrac A, Tung JK, Alves TC, Fang JS, Xie Y, Zhu J, Chen Z, De Smet F, Zhang J, Jin SW, Sun L, Sun H, Kibbey RG, Hirschi KK, Hay N, Carmeliet P, Chittenden TW, Eichmann A, Potente M, Simons M. FGF-dependent metabolic control of vascular development. Nature. 2017:545:224-8

18. Jiang S, Zhang LF, Zhang HW, Hu S, Lu MH, Liang S, Li B, Li Y, Li D, Wang ED, Liu MF. A novel miR-155/miR-143 cascade controls glycolysis by regulating hexokinase 2 in breast cancer cells. EMBO J. 2012;31:1985-98.

19. Londhe P, Yu PY, ljiri Y, Ladner KJ, Fenger JM, London C, Houghton PJ, Guttridge DC. Classical NF-kappaB metabolically reprograms sarcoma cells through regulation of hexokinase 2. Front Oncol. 2018:8:104.

20. Gupta P, Sheikh T, Sen E. SIRT6 regulated nucleosomal occupancy affects hexokinase 2 expression. Exp Cell Res. 2017:357:98-106.

21. Schuringa JJ, Wierenga AT, Kruijer W, Vellenga E. Constitutive Stat3, Tyr705, and Ser727 phosphorylation in acute myeloid leukemia cells caused by the autocrine secretion of interleukin-6. Blood. 2000;95:3765-70.

22. Wingender $E$, Chen X, Fricke E, Geffers R, Hehl R, Liebich I, Krull M, Matys V, Michael H, Ohnhauser R, Pruss M, Schacherer F, Thiele S, Urbach S. The 
TRANSFAC system on gene expression regulation. Nucleic Acids Res. 2001; 29:281-3.

23. Salvi M, Trashi E, Cozza G, Franchin C, Arrigoni G, Pinna LA. Investigation on PLK2 and PLK3 substrate recognition. Biochim Biophys Acta. 2012;1824: 1366-73.

24. Xu D, Yao Y, Lu L, Costa M, Dai W. Plk3 functions as an essential component of the hypoxia regulatory pathway by direct phosphorylation of HIF-1alpha. J Biol Chem. 2010;285:38944-50.

25. Xu D, Yao Y, Jiang X, Lu L, Dai W. Regulation of PTEN stability and activity by Plk3. J Biol Chem. 2010;285:39935-42.

26. Jonas $\mathrm{S}$, Izaurralde $\mathrm{E}$. Towards a molecular understanding of microRNAmediated gene silencing. Nat Rev Genet. 2015;16:421-33.

27. Hanahan D, Weinberg RA. Hallmarks of cancer: the next generation. Cell. 2011;144:646-74.

28. Levine AJ, Puzio-Kuter AM. The control of the metabolic switch in cancers by oncogenes and tumor suppressor genes. Science. 2010;330:1340-4.

29. Cairns RA, Harris IS, Mak TW. Regulation of cancer cell metabolism. Nat Rev Cancer. 2011;11:85-95

30. Straus DS. TNFalpha and IL-17 cooperatively stimulate glucose metabolism and growth factor production in human colorectal cancer cells. Mol Cancer. 2013;12:78.

31. Tambe Y, Hasebe M, Kim CJ, Yamamoto A, Inoue H. The drs tumor suppressor regulates glucose metabolism via lactate dehydrogenase-B. Mol Carcinog. 2016:55:52-63.

32. Bruno T, Valerio M, Casadei L, De Nicola F, Goeman F, Pallocca M, Catena V, lezzi S, Sorino C, Desantis A, Manetti C, Blandino G, Floridi A, Fanciulli M. Che-1 sustains hypoxic response of colorectal cancer cells by affecting Hif1alpha stabilization. J Exp Clin Cancer Res. 2017;36:32.

33. Patra KC, Wang Q, Bhaskar PT, Miller L, Wang Z, Wheaton W, Chandel N, Laakso M, Muller WJ, Allen EL, Jha AK, Smolen GA, Clasquin MF, Robey B, Hay N. Hexokinase 2 is required for tumor initiation and maintenance and its systemic deletion is therapeutic in mouse models of cancer. Cancer Cell. 2013;24:213-28

34. Mathupala SP, Ko YH, Pedersen PL. Hexokinase-2 bound to mitochondria: cancer's stygian link to the "Warburg effect" and a pivotal target for effective therapy. Semin Cancer Biol. 2009;19:17-24.

35. Lin L, Ding Y, Wang Y, Wang Z, Yin X, Yan G, Zhang L, Yang P, Shen $H$. Functional lipidomics: palmitic acid impairs hepatocellular carcinoma development by modulating membrane fluidity and glucose metabolism. Hepatology. 2017;66:432-48.

36. Yao A, Xiang Y, Si YR, Fan LJ, Li JP. PKM2 promotes glucose metabolism through a let-7a-5p/Stat3/hnRNP-A1 regulatory feedback loop in breast cancer cells. J Cell Biochem. 2019;120:6542-54

37. Helmke C, Becker S, Strebhardt K. The role of Plk3 in oncogenesis. Oncogene. 2016;35:135-47.

38. Zhang LF, Jiang S, Liu MF. MicroRNA regulation and analytical methods in cancer cell metabolism. Cell Mol Life Sci. 2017;74:2929-41.

39. Zhang GJ, Li JS, Zhou H, Xiao HX, Li Y, Zhou T. MicroRNA-106b promotes colorectal cancer cell migration and invasion by directly targeting DLC1. J Exp Clin Cancer Res. 2015;34:73.

40. Zheng L, Zhang Y, Liu Y, Zhou M, Lu Y, Yuan L, Zhang C, Hong M, Wang S, Li X. MiR-106b induces cell radioresistance via the PTEN/PI3K/AKT pathways and p21 in colorectal cancer. J Transl Med. 2015;13:252.

\section{Publisher's Note}

Springer Nature remains neutral with regard to jurisdictional claims in published maps and institutional affiliations.

Ready to submit your research? Choose BMC and benefit from:

- fast, convenient online submission

- thorough peer review by experienced researchers in your field

- rapid publication on acceptance

- support for research data, including large and complex data types

- gold Open Access which fosters wider collaboration and increased citations

- maximum visibility for your research: over $100 \mathrm{M}$ website views per year

At BMC, research is always in progress.

Learn more biomedcentral.com/submissions 